\title{
Optimized, Automated Striatal Uptake Analysis Applied to SPECT Brain Scans of Parkinson's Disease Patients
}

\author{
I. George Zubal, Michele Early, Olive Yuan, Danna Jennings, Kenneth Marek, and John P. Seibyl \\ Institute for Neurodegenerative Disorders, and Molecular Neuroimaging, LLC, New Haven, Connecticut
}

Reliable quantitative dopamine transporter imaging is critical for early and accurate diagnosis of Parkinson's disease (PD). Image quantitation is made difficult by the variability introduced by manual interventions during the quantitative processing steps. A fully automated objective striatal analysis (OSA) program was applied to dopamine transporter images acquired from PD subjects with early symptoms of suspected parkinsonism and compared with manual analysis by a trained image-processing technologist. Methods: A total of $101^{123} \mid-\beta-$ CIT SPECT scans were obtained of subjects recruited to participate in the QueryPD Study. Data were reconstructed and then analyzed according to a package of scripts (OSA) that reorients the SPECT brain volume to the standard geometry of an average scan, automatically locates the striata and occipital structures, locates the caudate and putamen, and calculates the background-subtracted striatal uptake ratio (V3"). The striatal uptake ratio calculated by OSA was compared with manual analysis by a trained image-processing technologist. Several parameters were varied in the automated analysis, including the number of summed transverse slices and the size and separation of the regions of interest applied to the caudate and putamen to determine the optimum OSA analysis. The parameters giving V3" with the closest correlation to the manual analysis were accepted as optimal. Results: The optimal comparison between the V3" obtained by the human analyst and that obtained by the automated OSA analysis yielded a correlation coefficient of 0.96 . Conclusion: Our optimized OSA delivers V3" evaluations that closely correlate with a similar evaluation manually applied by a highly trained image-processing technologist.

Key Words: PACS; neurology; SPECT; Parkinson's disease; analysis; automated

J Nucl Med 2007; 48:857-864

DOI: 10.2967/jnumed.106.037432

$\mathbf{P}$

arkinson's disease (PD) is characterized by the slowly progressive degeneration of nigrostriatal dopaminergic neurons as shown in Figure 1. Identification of the loss of dopaminergic neurons has led to effective symptomatic

Received Oct. 20, 2006; revision accepted Mar. 6, 2007.

For correspondence contact: I. George Zubal, PhD, Institute for Neurodegenerative Disorders, 60 Temple St., Suite 8, New Haven, CT 06510. E-mail: gzubal@indd.org

COPYRIGHT @ 2007 by the Society of Nuclear Medicine, Inc. therapy using dopamine replacement but does not prevent progressive neuronal loss and disability (1).

More accurate and objective assessment of disease onset and disease progression in individuals with PD is becoming increasingly important as neuroprotective and neurorestorative strategies for PD are developed and tested (2,3). These neuroprotective strategies further underscore the need for early and accurate diagnosis because typically about $50 \%$ of dopaminergic neurons have been lost by the time of symptom onset.

Although clinical diagnosis correlates well with pathologic diagnosis after long-term clinical follow-up (4), early in the course of PD the diagnoses may be difficult and often inaccurate. The most commonly mistaken diagnoses for PD include essential tremor, vascular parkinsonism, drug-induced parkinsonism, and Alzheimer's disease (5,6). In addition, subtle symptoms of motor slowness easily confused with parkinsonism may be found in 32\%-35\% of elderly subjects, often complicating diagnosis in this most common age group for PD $(7,8)$. More recently, the need for diagnosis to enable therapy even before symptoms are manifested, during the premotor period of $\mathrm{PD}$, has further highlighted the need for objective, accurate early diagnostic tools (9).

During the past decade, in vivo neuroimaging targeting the dopamine transporter (DAT) has emerged as a reliable diagnostic tool for PD and related parkinsonian syndromes. Numerous studies have demonstrated that DAT ligands and SPECT or PET identify individuals with PD and distinguish them from healthy subjects or individuals with movement disorders not associated with dopamine depletion, such as essential tremor, nonspecific gait disorders, drug-induced parkinsonism, and psychogenic parkinsonism (10-14). More recently, studies have further demonstrated that DAT imaging adds to diagnostic accuracy in patients with suspected PD at the earliest stages of illness $(15,16)$.

Reliable, rapid, easy-to-administer automated tools for quantitative analysis of DAT images would potentially improve diagnostic accuracy and enable more effective, widespread use of DAT imaging technology in clinical practice. Other investigators have developed methods to quantitate the uptake level of DATs by analyzing the associated SPECT 


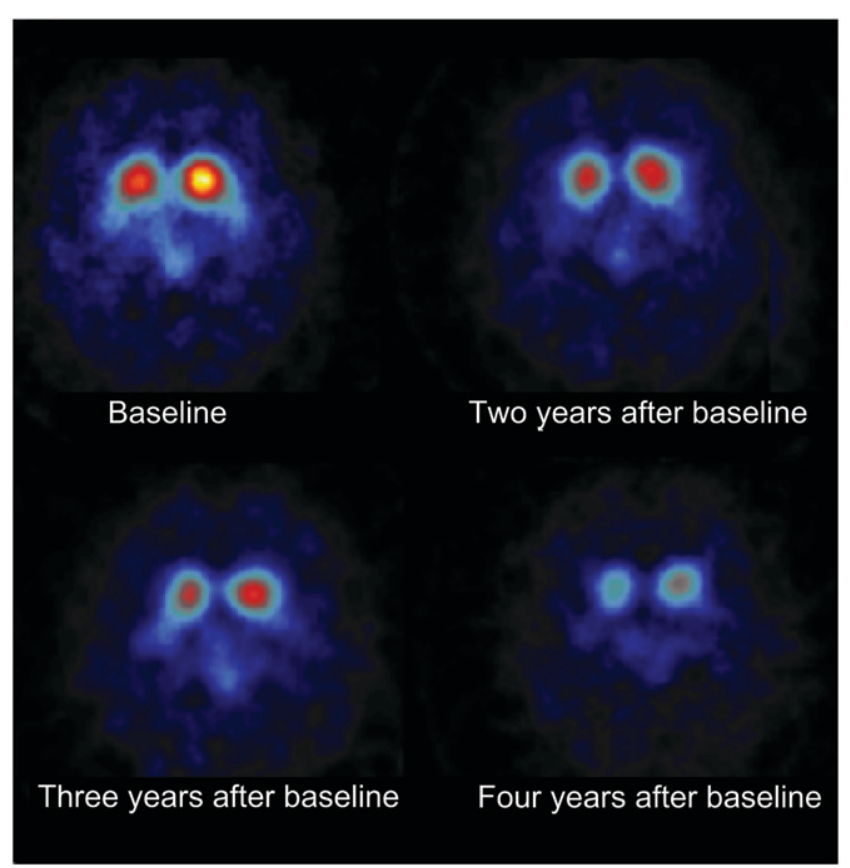

FIGURE 1. DAT imaging in PD patient followed for $4 \mathrm{y}$. Visually, there is decreased uptake of radiotracer in striatum, indicative of ongoing loss of presynaptic dopaminergic function. Although the loss is easily noted, it is difficult to determine clinical meaningfulness of this extent of signal change without quantitation.

scans (17-21). In this study, we tested an automated DATimaging objective striatal analysis (OSA) program specifically for ${ }^{123} \mathrm{I}-\beta$-CIT in a large clinical cohort and compared the results with a well-established manual analysis by a trained image-processing technologist.

\section{MATERIALS AND METHODS}

The clinical cohort evaluated in this study was recruited as part of the Query-PD Study (15), a study to determine the diagnostic accuracy of DAT imaging in subjects identified by community neurologists as suspected of having, but not yet diagnosed with, PD or parkinsonian syndrome. All subjects signed informed consent and were clinically evaluated at Molecular NeuroImaging, LLC, by a movement disorders expert. Subjects met the following inclusion criteria: age greater than $21 \mathrm{y}$, any parkinsonian or extrapyramidal symptoms as determined by the primary neurologist, parkinsonian symptoms for less than $2 \mathrm{y}$, and no significant abnormalities on screening laboratory studies, including complete blood cell count, Chem-20, and urinalysis.

All subjects underwent ${ }^{123}$ I- $\beta$-CIT DAT imaging at Molecular NeuroImaging, LLC, using a previously described and operationalized image acquisition (16). All patients received supersaturated potassium iodide solution ( $800 \mathrm{mg}$ orally) before tracer injection. After injection of $370 \pm 57 \mathrm{MBq}(10.0 \pm 1.6 \mathrm{mCi})$, projection data were obtained in a $128 \times 128$ matrix on a Picker Prism 3-head camera (Philips Medical Systems) mounted with fanbeam collimators, by acquiring 120 angles over $40 \mathrm{~min}$. The data were reconstructed using the manufacturer-supplied (Odyssey VP 8.5A5) standard filtered backprojection algorithm and applying a postreconstruction 3-dimensional Butterworth smoothing filter (order, 6; cutoff, 0.24 cycles per pixel). With the radius of rotation set to
$15 \mathrm{~cm}$, this process results in tomographic resolutions of $2.1 \mathrm{~mm}$ in the $x$ - and $y$-directions and $3.56 \mathrm{~mm}$ in the $z$-direction. Attenuation was corrected using the Chang zero-order correction (22), applying a linear attenuation coefficient of $\mu=0.11 \mathrm{~cm}^{-1}$. The neuroimaging personnel were unaware of any clinical information.

For quantitation of DAT SPECT data, stringent techniques involving a highly operationalized series of steps were performed by an experienced image processor. These manual steps, using the manufacturer-supplied processing software, included, first, 2-dimensional Butterworth filtration (order, 10; cutoff, 0.24) of the 120 projection images acquired for each scan; second, reconstruction using filtered backprojection and a simple ramp filter; third, manual reorientation of the reconstructed imaging volume along the canthomeatal line, determined from external radioactivity-containing fiducial markers placed on the subject's skin; fourth, identification of axial slices with striatal activity, summation of the specified number of slices, and application of a Chang attenuation correction; fifth, placement of a regional template on the left and right caudate and putamen and the occipital background region; and sixth, extraction of count density data for determination of regional striatal V3" as indicated in the following equation: V3" = (regional striatal count density - occipital count density)/occipital count density.

After completion of the predefined reconstruction, manual image processing included several steps. The canthomeatal line and the mid plane were visually determined between the 2 hemispheres so as to rotate them into a horizontal and a vertical orientation, respectively. Once reoriented, 8 transverse slices were visually selected as representing the centralmost slices containing the striata. These selected slices were added together to create a single slice for region-of-interest (ROI) placement. The imageprocessing platform has a predefined template containing the attenuation correction ellipse and associated ROIs corresponding to the expected position of the caudate and putamen. After the attenuation ellipse was placed onto the transverse slice, the striatal ROIs could be manually adjusted until the operator decided that they were optimally overlaid onto the caudate and putamen. Similarly, a large semicircular ROI could be repositioned until the operator decided that it best measured the distribution of counts found in the occipital lobes. Once these ROIs were finally positioned, V3" was calculated. The positioning of the attenuation ellipse and ROIs over the striatal and occipital structures was based on uptake intensities seen on the transverse images of the ${ }^{123}$ I- $\beta$-CIT SPECT scan. Because no accompanying anatomic scans (CT or MRI) are routinely acquired for subjects undergoing ${ }^{123}$ I- $\beta$-CIT SPECT, the operator relied on functional information alone when placing these ROIs. Fortunately, even for patients with advanced disease, the caudate demonstrated a relatively high ratio of tracer uptake, compared with the surrounding nonspecific background, and could easily be identified.

\section{Automated Image Processing}

A noninteractive OSA software package was developed for application to the already-reconstructed and attenuation-corrected SPECT brain volumes and accomplished the third, fourth, fifth, and sixth steps above. These Matlab scripts run under Matlab version 6.5.1 (The MathWorks, Inc.) and perform the imageprocessing steps outlined below.

\section{Reorientation of the Reconstructed Imaging Volume}

The patient's SPECT scan was reoriented by adoption of a stylized average scan, which represented an averaged brain volume 
of 26 randomly selected subjects (13 healthy and 13 with PD). This average scan was manually reoriented into a position in which the canthomeatal line lay parallel to the horizontal planes within the imaging matrix. Once this standard orientation was available and stored, all other subjects' scans were geometrically registered to this average scan using an implementation of the mutual information algorithm (23). Figure 2 shows the display screen for this registration process. The left half of this figure shows the stylized average scan, in which a sum of all sagittal, transverse, and coronal slices is displayed in the top left, bottom left, and bottom right panels, respectively. The right half of this figure shows a similarly displayed example of the subject's summed sagittal, transverse, and coronal views. The green, blue, and red contours overlaid onto the subject's images correspond to the isocontour lines transferred from the average scan and help to show the accuracy of the registration.

The rationale for registering the patient's scan to the average scan is to ensure that the patient's brain volume is oriented in a standard position, that is, to correct for any rotation around the $x$-, $y$-, or z-axis of the patient's head within the imaging camera. This correction is important because the subsequent location of striata and placement of ROIs (striatal and background) can be influenced by variations in the pitch, yaw, and roll orientations of the head. However, to optimize registration between the patient's scan and the average scan (despite variation in individual head size), one needs to apply a scaling. Hence, the average scan is scaled (but not rotated or translated) independently in the $x$-, $y$-, and $z$-axes when the patient's scans are registered to the average scan, yielding an optimal registration of the patient's scan with the orientation of the average scan, without scaling or warping the patient's scan (which could potentially introduce additional interpolation errors later in the quantitation).

\section{Identification of Striata}

Identification of the striata is fairly simple. Even in advanced disease, the target-to-background ratio is high and the striata are small in relation to the whole brain. The 2-step process of locating the centers of the 2 striata within the patient's brain volume is shown in Figure 3.

The location of the horizontal central slice passing through the center of the striata is shown in the left half of Figure 3. By calculating the top-to-bottom profile of the summed intensities in the sagittal view, one can easily identify the location of the central slice through the striata as the maximum along this profile. The small white panel shows the graph of this summed profile, and the green arrow points along the central slice to the location of the striata. The red lines above and below show the selection of $N$ transverse slices for subsequent processing (in this example, $N=$ 9). The right half of Figure 3 shows the same subject's brain volume when the intensities above and below the selected $N$ slices are set to zero. Maxima along the summed profiles of the sagittal and coronal images localize the position of the 2 striata. The green arrows point back from the maxima along the profiles to the location of the 2 striata.

All subsequent processing from this point on is applied to these selected $N$ (summed) transverse slices. Selecting the optimal value for $N$ is described in the section on parameter optimization.

\section{Measurement of Head Size and Placement of ROIs}

To properly place the ROIs on the caudate, putamen, and occipital lobes, one must calibrate each subject's relative head size. The calibration method is shown in the left half of Figure 4, in which a summed profile is calculated along the anterior-toposterior direction of the $\boldsymbol{N}$ selected slices and is displayed in the white panel.

The peak corresponding to the striata appears superimposed on a fairly well defined plateau of nonspecific activity distributed throughout the brain. The edge of the occipital lobe is taken as the $50 \%$ intensity threshold found on the posterior portion of this plateau profile. The location of the striata and edge of the occipital lobe are shown with red lines drawn on the sagittal and transverse image displays and on the profile in the white panel. The pixel distance between these red lines is the head size.

The head size of the average scan is 55 pixels. When subjects are analyzed, the size $(s)$ and separation $(\boldsymbol{d})$ of the ROIs to be applied on the caudate and putamen, as well as the size of the occipital lobe ROI, are linearly scaled in proportion to each subject's head size.

Once the scaling for the ROIs is determined, as shown in the right half of Figure 4, a larger caudate ROI (green circle) is moved within the $\mathrm{N}$-summed slices image until the ROI yields a maximum value of total counts; this is done for both the left and the right striatum. The small vertical and horizontal arrows depict this searching algorithm. Within each green circle, a smaller caudate ROI (red circle) is placed at its center and is used for the count density measurement of each caudate. Given a default separation of the caudate to putamen, $\boldsymbol{d}$, the putamen ROI (yellow circle) orbits around the caudate ROI at the appropriately scaled distance until the total counts within the ROI yield a maximum; the curved yellow arrows depict this orbiting, searching algorithm.

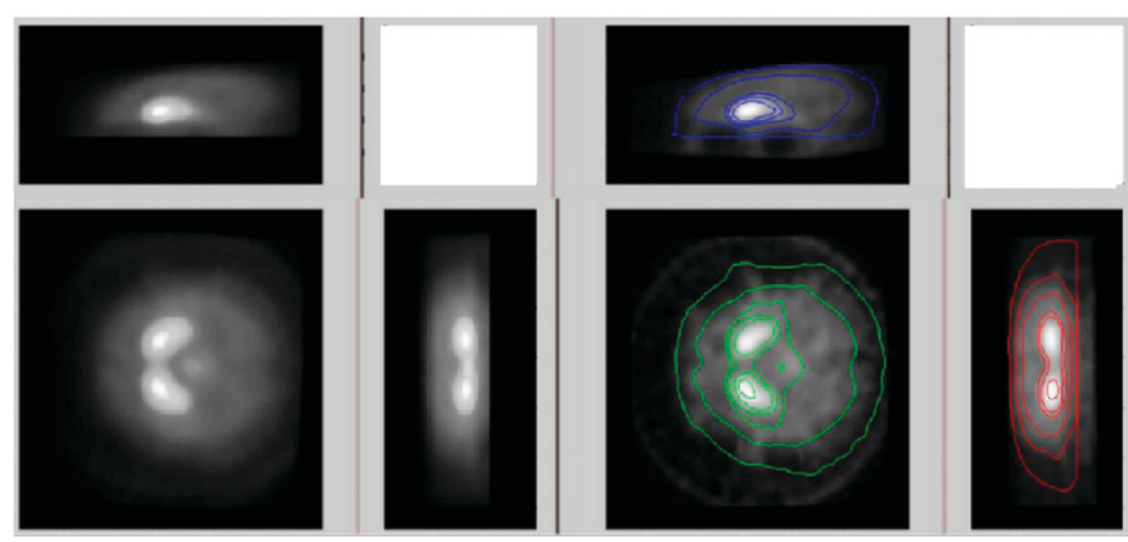

FIGURE 2. Display showing example patient's brain scan (right half of figure) being registered to standard average scan (left half of figure). 
FIGURE 3. Display showing selection of horizontal striatal midplane (left half of figure) and subsequent localization of left and right striatum (right half of figure), both based on location of maxima in either sagittal or coronal profiles.

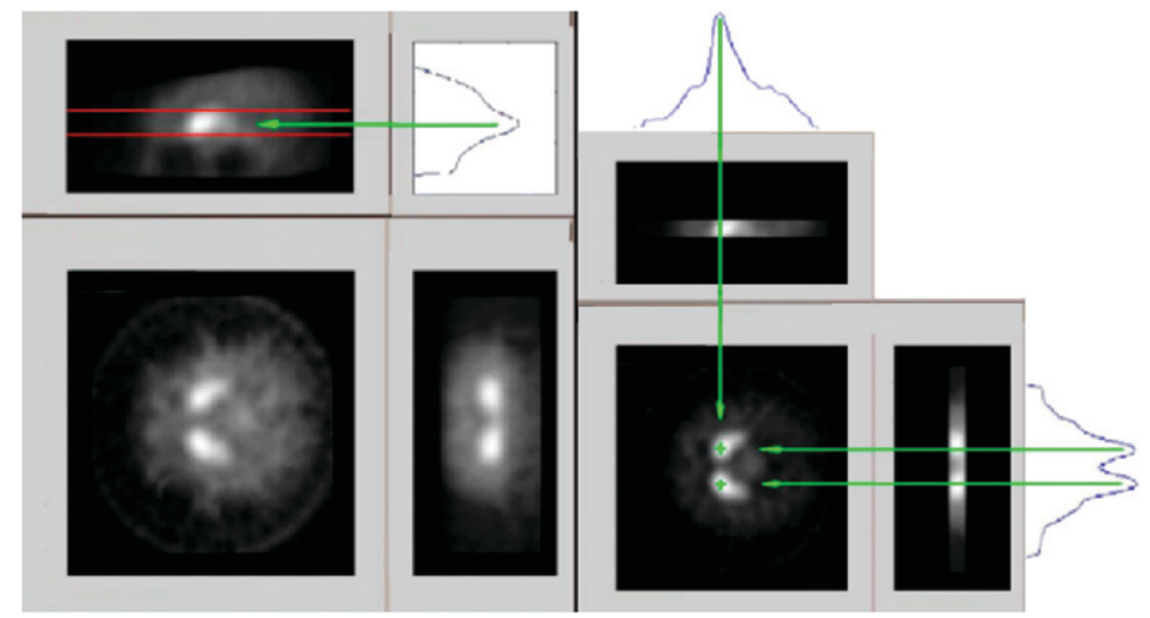

The default occipital ROI (large red truncated annulus) is scaled equally in the $x$ - and $y$-directions and is placed just inside the edge of the occipital lobe.

The regional striatal count density is calculated as the average of the 2 caudate and 2 putamen ROI count densities, the occipital count density is calculated from the occipital ROI, and V3" is calculated according to Equation 1.

\section{Optimizing the Parameters for Analysis}

The gold standard for testing the accuracy of the automated OSA program is the V3" manually obtained by a trained, highly experienced image-processing technologist. However, it was not known which values of $\boldsymbol{N}, \boldsymbol{s}$, and $\boldsymbol{d}$ would correlate best with the human analysis. To determine the optimal values for $N, s$, and $d$, all 101 patients were batch analyzed, by which the parameter $N$ took on values of $4,5,7,9,11,13,15$, and 17 (slices); the parameter $\boldsymbol{s}$ took on values of 1,2 , and 3 (pixels); and the parameter $\boldsymbol{d}$ took on values of $8,9,10,11$, and 12 (pixels); that is, all 101 patients were analyzed 120 times (each time with a different set of $\boldsymbol{N}, \boldsymbol{s}$, and $\boldsymbol{d}$ parameters) and then compared with manual analysis by calculation of the linear regression slope and its associated $R$ value.

\section{RESULTS}

Figure 5 shows a portion of the 120 evaluations performed using OSA. In this section of the analysis, the parameters $N$, $\boldsymbol{s}$, and $\boldsymbol{d}$ were varied between 7 and 13, between 1 and 4, and between 9 and 12, respectively (plotted along the horizontal axis). For each set of these parameters, $101 \mathrm{~V} 3$ " evaluations were compared with the manual analysis, yielding an $R$ value (plotted along the vertical axis). The cyclic oscillations of the $R$ values demonstrated that as the values of $N, s$, and $d$ were varied, the correlation between the manual and automated determinations of V3" for this group of subjects changed.

The accuracy of the OSA analysis was optimal when $N$, $\boldsymbol{s}$, and $\boldsymbol{d}$ took on values of 9,1 , and 8 , respectively. The scatter plot for this case is shown in Figure 6. The interpretation of $s=1$ is the use of a $3 \times 3$ pixel matrix ROI (i.e., a central pixel \pm 1 neighbor pixel in each direction).

The precision of the OSA analysis was optimal when $N$, $\boldsymbol{s}$, and $\boldsymbol{d}$ took on values of 13,0 , and 10 , respectively, corresponding to the maximum $R$ value in Figure 5. The scatter plot for this case is shown in Figure 7. The interpretation of $s=0$ is the use of a single-pixel ROI.

Accuracy (measured by the linear regression slope) and precision (measured by the $R$ value) changed as the combination of parameters $\boldsymbol{N}, \boldsymbol{s}$, and $\boldsymbol{d}$ took on various values. Figure 8 shows this variation when the regression slope and $R$ value were plotted as a function of $N, s$, and $d$. Here, $N, s$,
FIGURE 4. Display showing automatic measurement of head size (left half of figure), whereby distance from midpoint between the 2 striata to edge of occipital lobes is used to scale subsequent ROI placement. Right half of figure shows placement of caudate ROls (small red circles), putamen ROls (small yellow circles), and occipital ROI (large truncated annulus).

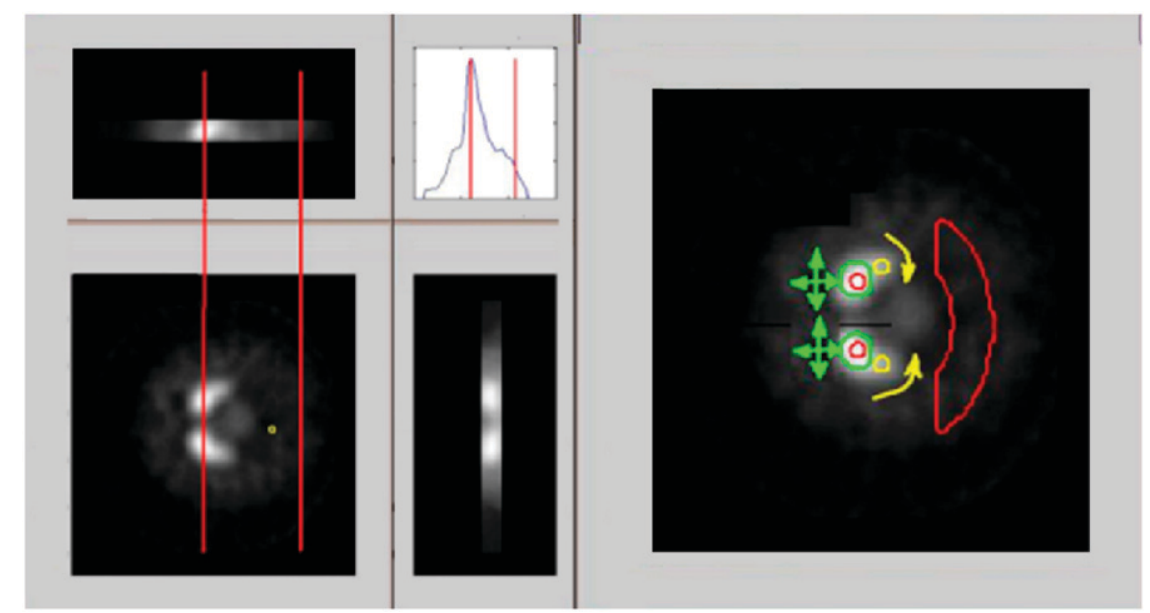




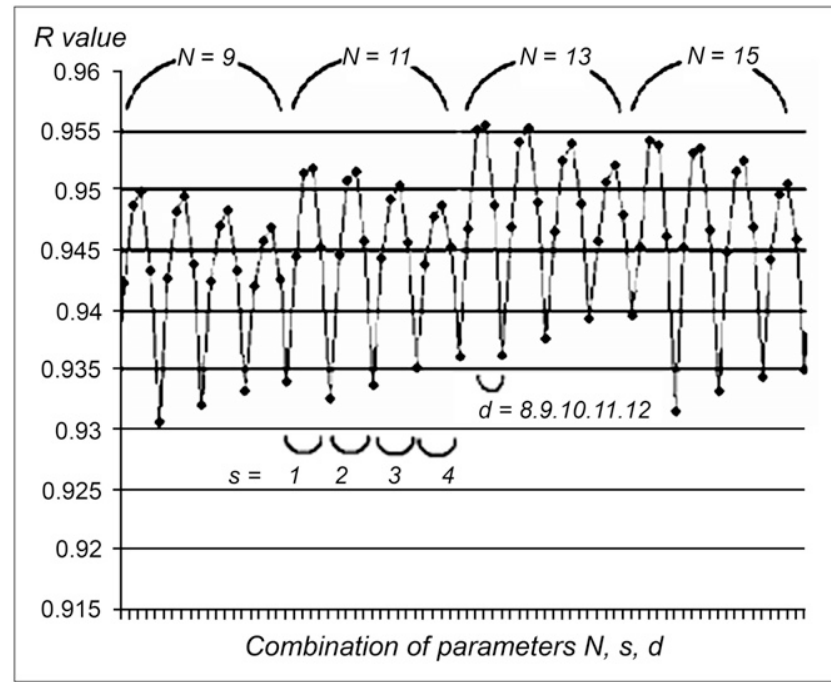

FIGURE 5. Graph of $R$ value versus combination of $\boldsymbol{N}, \mathbf{S}$, and $\boldsymbol{d}$ parameters used by OSA. $R$ value comes from comparison of OSA V3" values to those obtained by experienced image processing researcher. Values of $\boldsymbol{N}$ vary from 9 to 15 from left to right along axis; values of $s$ change from 1 to 4 and cycle more slowly from left to right than do values of $\boldsymbol{d}$, which cycles more quickly through values of 8 to 12 .

and $\boldsymbol{d}$ vary from values of 7,0, and 8, respectively, at the left side of the horizontal axis to values of 17,1 , and 9, respectively, at the right side of the axis. Because optimal slope and $R$ value would be equal to 1.0, it is interesting to note that the values of $R$ and the slope cross each other at a point corresponding to $\boldsymbol{N}, \boldsymbol{s}$, and $\boldsymbol{d}$ values of 9,1 , and 8 , respectively (the parameters that yielded the most accurate results), and that the plot of the slope contains 6 repeating cycles that correspond to the selection of increasing $N$ values. Increasing the $N$ values had the strongest influence on decreasing the accuracy of OSA.

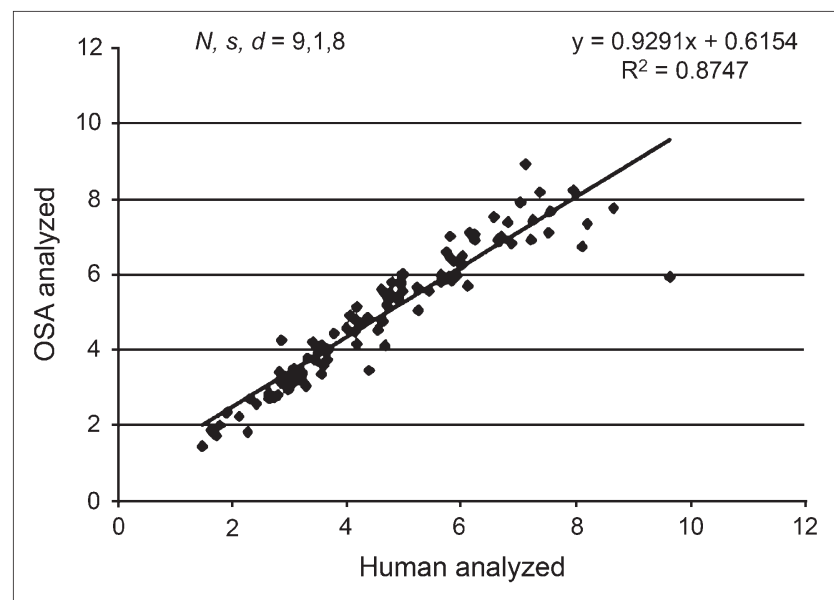

FIGURE 6. Scatter plot of OSA V3" evaluations compared with those of experienced researcher for OSA parameters $\mathbf{N}, \mathbf{s}$, and $\boldsymbol{d}$ set to 9,1 , and 8 , respectively. These parameters yielded optimal slope of 0.93 and demonstrated best accuracy for OSA analysis.

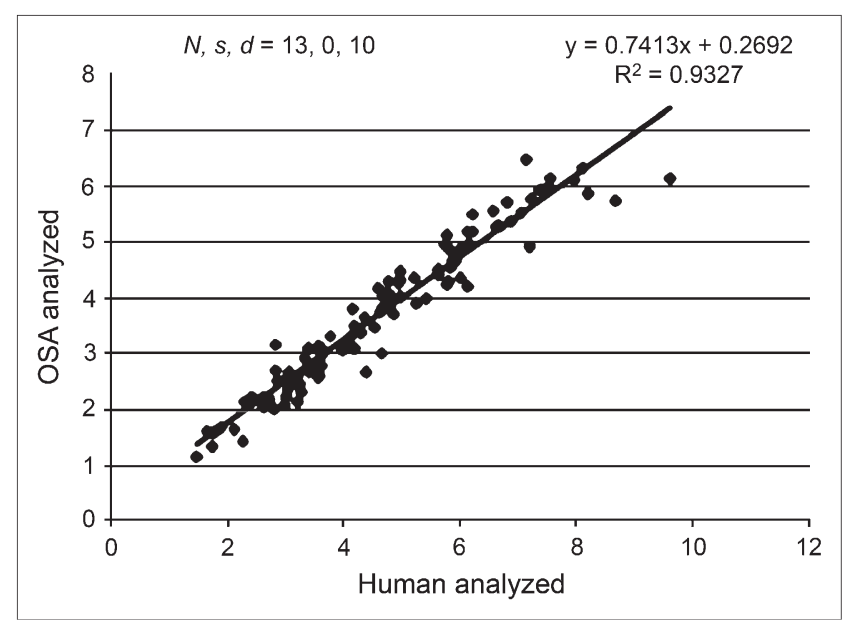

FIGURE 7. Scatter plot of OSA V3" evaluations compared with those of experienced researcher for OSA parameters $\mathbf{N}, \mathbf{s}$, and $\boldsymbol{d}$ set to 13,0 , and 10 , respectively. These parameters yielded optimal $R$ value of $0.96\left(R^{2}=0.93\right)$ and demonstrated best precision for OSA analysis.

\section{DISCUSSION}

Our optimized, automated analysis (OSA) delivered ${ }^{123} \mathrm{I}$ $\beta$-CIT striatal V3" values for 101 patients with suspected parkinsonism that strongly correlated $(r=0.96)$ with the same evaluations conducted by a highly trained image-processing researcher. OSA provides a rapid, efficient, flexible, and reliable tool for quantitative DAT imaging. One particular advantage of OSA is that the program can be exported to

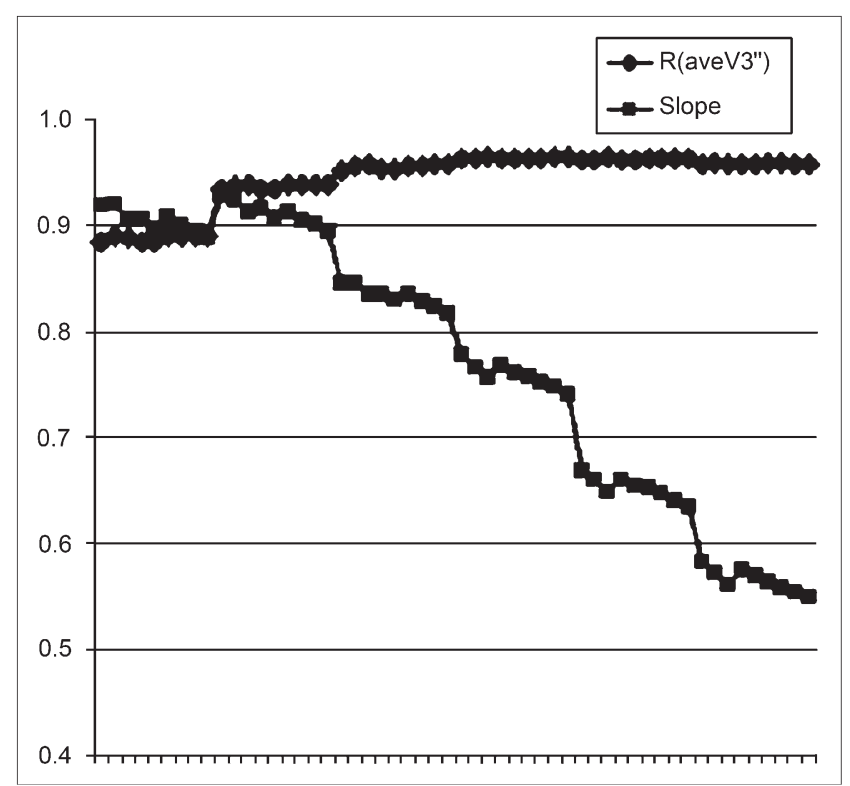

FIGURE 8. Plot of both $R$ value and regression slope vs. combination of $\boldsymbol{N}, \mathbf{S}$, and $\boldsymbol{d}$ parameters used by OSA. Parameters along horizontal axis are similar to those in Figure 5 in that, here, values of $\boldsymbol{N}$ vary from 3 to 17 from left to right along axis; values of $\boldsymbol{s}$ change from 1 to 4 and cycle more slowly from left to right than do values of $\boldsymbol{d}$, which cycles more quickly through values of 8 to 12 . 
multiple imaging sites, where the identical analysis is conducted as it would be by a central image-processing laboratory. The availability of an automated imaging-analysis program is critical to the widespread use of DAT imaging, particularly at sites with little experience in analyzing DAT SPECT images or DAT radiotracers involving multiple dynamic acquisitions after radiotracer injection.

DAT imaging for PD diagnosis is commercially available in most European countries and is currently under development in the United States. Accurate and reliable quantitation of DAT imaging outcomes is critical to early diagnosis, and the need for earlier PD diagnosis to allow earlier treatment is growing. It is well known that PD has a prolonged presymptomatic period during which neurodegeneration occurs but symptoms have not yet manifested. Imaging offers the best opportunity to accurately push back the diagnosis to the presymptomatic period (24). Although imaging is unlikely to be used as a primary screen for PD because of cost, coupling of DAT imaging with more widely available PD biomarkers may effectively identify subjects who might be presymptomatic (25). As DAT imaging is used in increasing numbers of subjects and imaging sites, OSA would provide both a reliable and a practical method to ensure accurate quantitation of imaging outcomes.

OSA is fully automated, that is, after the file names for the attenuation-corrected reconstructed brain volumes have been selected, all subsequent processing is accomplished without any manual intervention and is completed within 2 min (running on Windows XP [Microsoft] with a $1-\mathrm{GHz}$ processor), $90 \%$ of which time is needed for 3-dimensional registration to the average scan. For the clinical cohort in this study, using a simple batch script, 101 subjects were reanalyzed within $3.5 \mathrm{~h}$. This brief processing time allows further analyses for evaluating the effect of attenuation correction, scatter correction, iterative or filtered backprojection reconstruction parameters, and other variables on the resulting V3" by varying reconstruction and correction methods for the same original camera data acquisitions.

Our aim in this development was to duplicate the previously completed ${ }^{123}$ I- $\beta$-CIT striatal V3" manual analysis for this clinical cohort (15). Therefore, the optimal parameters $(\boldsymbol{N}, \boldsymbol{s}$, and $\boldsymbol{d})$ for the OSA program were selected so as to most closely correlate with the manual analysis obtained by our image-processing researcher. However, these optimized parameters may not be the best for applying OSA to different clinical cohorts with more severe loss of DAT or for monitoring the change in DAT loss resulting from disease progression. For example, optimizing the parameters for a group of healthy subjects should probably yield the smallest group change in V3" for repeated studies over several weeks. By a similar argument, optimal parameters applied to a group of advanced-PD patients would be expected to yield the largest group change in V3" for repeated studies over several months. Theoretically, the optimal parameters for applying OSA would simultaneously optimize the results for both these example groups. OSA is a flexible tool that can be customized to measure DAT loss at PD diagnosis or the change in DAT with PD progression.

Optimal parameters for OSA may also depend on technical issues such as camera and collimator type, implementation of attenuation or scatter correction, and selection of a postreconstruction smoothing filter that may merit optimizing the $\boldsymbol{N}$, $\boldsymbol{s}$, and $\boldsymbol{d}$ parameters for a different set of site-specific imaging and reconstruction protocols. However, as is the case for several other packaged analysis programs $(17,19-21)$ that report the number of analysis slices and placement of ROIs, one often assumes that technical issues concerning the acquisition and reconstruction of image data can be considered "acceptably similar" and, thus, not require optimization for each camera. This assumption is reasonable, because optimization of the parameters is a daunting, time-consuming task; the resulting optimized parameters would perhaps be similar (if not identical) to the original ones; and camera manufacturers have evolved toward camera and reconstruction characteristics that in most cases are similar and reproducible.

Other investigators have focused on brain image-processing packages $(17-21,26)$. In its application, OSA most closely resembles the automated analysis package reported by Koch et al $(20,21)$. Although both software analysis packages ultimately focus on calculating V3" as described in Equation 1, the 2 methods differ in their philosophies on image manipulation and ROI-based uptake measurements.

When repositioning each subject's reconstructed SPECT brain volume into a standard orientation, OSA applies only rigid-body translation and rotation. Independent $x$-, $y$-, and $z$-scaling is applied only to the average scan for optimizing the subject's registration accuracy to this standard orientation. No nonlinear warping is applied to the subject's data, because so doing may add interpolation errors. When SPECT scans are repeated on the same subject, subsequent scans are registered to the same subject's first scan (already registered to the average scan) instead of to the average scan. We believe this method is beneficial, especially when changes in V3" are calculated in following disease progression.

With regard to the average scan used for reorienting each subject's SPECT scan, 26 random scans were intentionally selected so as to yield an "averaged" representation of the location of the striata within an "average" subject who would be imaged in our institute. Given such an averaged geometry, the intent of the registration process is to remove pitch, roll, and yaw positioning differences between subjects; that is, the registration process is meant to orient each subject reproducibly and standardly within the imaging camera. Furthermore, we emphasize that although uptake in the striata is more intense, the remaining volume of the brain (demonstrating nonspecific uptake) plays a larger role in determining the final orientation of a subject's brain scan. Because the calculation of best fit relies on minimizing variances between the average scan and the subject's scan, the smaller number of voxels in the striata diminishes the importance of these structures for the best-fit calculation, and 
the larger volume of the remaining gray matter plays a larger role in optimizing the registration. These factors allow us to use a single, stylized average scan for registering either relatively disease-free subjects or subjects exhibiting advanced, progressed disease. Additionally, this registration process is not associated with any localization of the striatal structures. The localization of striata is a totally independent process.

Another difference from other automated approaches is that OSA does not apply a rigid array of predefined ROI templates to the internal substructures of the brain. Instead, for the caudate and putamen, the most intense pixel in the caudate is located and the neighborhood of this location is used for evaluating caudate uptake. We have found that either for relatively minimal or for severely progressed PD, the most intense area of tracer uptake closely corresponds to the center of the caudate. The placement of the ROI for the putamen is similarly found by searching at a fixed radial distance from the caudate until the ROI yields a maximum intensity. The scaled occipital ROI is placed at a consistent distance from the edge of the posterior brain. These ROIs do not assume a predefined volume for each structure and, in the case of the caudate and putamen, measure the maximum uptake of the structure. For cardiac applications $(27,28)$, a method of measuring the hottest pixel with radiotracer techniques has been shown to be a reproducible and accurate way to evaluate physiologic function and is less prone to partial-volume effects resulting from the complex spatial geometry of the striatum or atrophic changes.

Although the most precise results in the comparison of OSA to manual analysis delivers an $R$ value of 0.97 , not all the variability should be attributed to the automated method of OSA for placing ROIs. In the case of manual analysis, projection data were reconstructed, attenuation-uncorrected transverse slices containing the striata were summed, and then an ellipse was placed onto the summed slice to apply the Chang correction for attenuation correction using our image-processing platform. In the case of OSA analysis, projection data were reconstructed with attenuation correction (using the reconstruction package of the camera manufacturer) and then made available for subsequent OSA analysis. Hence, the data used for manual and OSA analysis were not attenuation-corrected using the same ellipse placement. This discrepancy in ellipse placement rests on the operational characteristics of the workstation and camera computer used for reconstruction and analysis. As we have documented previously (29), when compared with heterogeneous attenuation corrections, placement of the attenuation correction ellipse for homogeneous attenuation correction can introduce 5\%-10\% error in internal brain structures and, therefore, can degrade the correlation coefficient between the OSA and manual analysis presented here.

Although accuracy and precision were independently optimized in this analysis, clearly, the best method for reporting the results for the 101 subjects studied here would be to apply the parameters yielding optimal precision (i.e., $r=0.9658$ with linear regression line $y=0.7413 x+0.2692$ ) and then to apply the linear regression as a correction to obtain both the most precise and the most accurate V3" values.

To evaluate the test/retest manual analysis of V3" in our institute, 2 of our image-processing technologists reanalyzed 20 randomly selected scans. Although the identical original SPECT dataset was used as the starting point, the $\mathrm{SD}$ in reevaluating the V3" for these scans showed a $6 \%$ variability. This reflects the subjectivity involved in manual analysis of these scans. OSA compared favorably: Reanalysis of the same original dataset yielded a variability of $0 \%$.

DAT imaging has been used in several clinical studies to monitor disease progression and assess possible neuroprotective drugs $(30,31)$. OSA would be an extraordinarily valuable tool for these studies, enabling objective analysis of imaging data potentially acquired at multiple sites. In further studies, we plan to validate OSA in existing clinical cohorts of PD progression to fully establish the optimal OSA parameters for both PD diagnosis and PD monitoring.

Finally, we believe our development of this automated image-processing technique contributes to a recent trend within nuclear medicine-a trend toward removing subjectivity from the analysis and interpretation of image data. Software packages for the automated processing and analysis of cardiac function images first appeared in the 1990s (32-34). More recently, automated techniques have been applied to brain images $(17,19-21)$. A software package for automated renal function has also been recently reported (35). We believe these developments enhance the clinical power of imaging studies in that they reduce the variability of quantitative results obtained between image-processing technologists and image-acquisition sites. Reduced variability helps to reduce the number of subjects needed to investigate new diagnostic and therapeutic agents and delivers more consistent and reproducible clinical evaluations.

\section{CONCLUSION}

Our optimized OSA delivers V3" evaluations that closely correlate with a similar evaluation manually applied by a highly trained image-processing technologist.

\section{REFERENCES}

1. Seibyl JP. Imaging studies in movement disorders. Semin Nucl Med. 2003;33: 105-113.

2. Schapira AH, Olanow CW. Neuroprotection in Parkinson disease: mysteries, myths, and misconceptions. JAMA. 2004;291:358-364.

3. Shoulson I. Experimental therapeutic of neurodegenerative disorders: unmet needs. Science. 1998;282:1072-1074.

4. Hughes AJ, Daniel SE, Ben-ShlomoY, Lees AJ. The accuracy of diagnosis of parkinsonian syndromes in a specialist movement disorder service. Brain. 2002;125:861-870.

5. Meara J, Bhowmick BK, Hobson P. Accuracy of diagnosis in patients with presumed Parkinson's disease. Age Ageing. 1999;28:99-102.

6. Quinn N. Accuracy of clinical diagnosis in early Parkinson disease. Arch Neurol. 2001;58:316-317. 
7. Bennett DA, Beckett LA, Murray AM, et al. Prevalence of parkinsonian signs and associated mortality in a community population of older people. $N$ Engl J Med. 1996;334:71-76.

8. Richards M, Stern Y, Mayeux R. Subtle extrapyramidal signs can predict the development of dementia in elderly individuals. Neurology. 1993;43:2184-2188.

9. Langston JW. The Parkinson's complex: parkinsonism is just the tip of the iceberg. Ann Neurol. 2006;59:591-596.

10. Booij J, Habraken JB, Bergmans P, et al. Imaging of dopamine transporters with iodine-123-FP-CIT SPECT in healthy controls and patients with Parkinson's disease. J Nucl Med. 1998;39:1879-1884.

11. Fischman AJ, Bonab AA, Babich JW, et al. [(11)C, (127)I] Altropane: a highly selective ligand for PET imaging of dopamine transporter sites. Synapse. 2001; 39:332-342

12. Mozley PD, Schneider JS, Acton PD, et al. Binding of $\left[{ }^{99 \mathrm{~m}} \mathrm{Tc}\right] \mathrm{TRODAT}-1$ to dopamine transporters in patients with Parkinson's disease and in healthy volunteers. J Nucl Med. 2000;41:584-589.

13. Parkinson Study Group. A multicenter assessment of dopamine transporter imaging with Dopascan ${ }^{\mathrm{TM}} / \mathrm{SPECT}$ in parkinsonism. Neurology. 2000;55:15401547.

14. Seibyl JP, Marek KL, Quinlan D, et al. Decreased single-photon emission computed tomographic $\left[{ }^{123} \mathrm{I}\right]$ beta-CIT striatal uptake correlates with symptom severity in Parkinson's disease. Ann Neurol. 1995;38:589-598.

15. Jennings DL, Seibyl JP, Oakes D, et al. $\left[{ }^{123} \mathrm{I}\right] \beta-\mathrm{CIT}$ and single-photon emission computed tomographic imaging vs clinical evaluation in Parkinsonian syndrome: unmasking an early diagnosis. Arch Neurol. 2004;61:1224-1229.

16. Catafau AM, Tolosa E. Impact of dopamine transporter SPECT using ${ }^{123} \mathrm{I}-$ Ioflupane on diagnosis and management of patients with clinically uncertain Parkinsonian syndromes. Mov Disord. 2004;19:1175-1182.

17. Buchert R, Berding G, Wilke F, et al. IBZM tool: a fully automated expert system for the evaluation of IBZM SPECT studies. Eur J Nucl Med Mol Imaging. 2006;33:1073-1083.

18. Morton RJ, Guy MJ, Clauss R, Hinton PJ, Marshall CA, Clarke EA. Comparison of different methods of DatSCAN quantification. Nucl Med Commun. 2005;26: 1139-1146.

19. Radau PE, Linke R, Slomka PJ, Tatsch K. Optimization of automated quantification of ${ }^{123}$ I-IBZM uptake in the striatum applied to parkinsonism. J Nucl Med. 2000;41:220-227.

20. Koch W, Hamann C, Welsch J, et al. Is iterative reconstruction an alternative to filtered backprojection in routine processing of dopamine transporter SPECT studies? J Nucl Med. 2005;46:1804-1811.
21. Koch W, Radau P, Hamann C, Tatsch K. Clinical testing of an optimized software solution for an automated, observer-independent evaluation of dopamine transporter SPECT studies. J Nucl Med. 2005;46:1109-1118.

22. Chang L. A method for attenuation correction in computed tomography. IEEE Trans Nucl Sci. 1987;NS-25:638-643.

23. Woods RP, Cherry SR, Mazziotta JC. Rapid automated algorithm for aligning and reslicing PET images. J Comput Assist Tomogr. 1992;16:620-633.

24. DeKosky S, Marek K. Looking backward to move forward: early detection of neurodegenerative disorders. Science. 2003;302:830-834.

25. Stern MB. The preclinical detection of Parkinson's disease: ready for prime time? Ann Neurol. 2004;56:169-171.

26. Ishii K, Kono AK, Sasaki H, et al. Fully automatic diagnostic system for earlyand late-onset mild Alzheimer's disease using FDG PET and 3D-SSP. Eur J Nucl Med Mol Imaging. 2006;33:575-583.

27. Hitzel A, Manrique A, Cribier A, Vera P. Diagnostic value of Tl-201 lung uptake is dependent on measurement method. J Nucl Cardiol. 2001;8:332-338.

28. Morel O, Pezard P, Furber A, et al. Thallium-201 right lung/heart ratio during exercise in patients with coronary artery disease: relation to thallium-201 myocardial single-photon emission tomography, rest and exercise left ventricular function and coronary angiography. Eur J Nucl Med. 1999;26:640-646.

29. Rajeevan N, Zubal IG, Ramsby SQ, Zoghbi SS, Seibyl J, Innis RB. Significance of nonuniform attenuation correction in quantitative brain SPECT imaging. J Nucl Med. 1998;39:1719-1726.

30. Parkinson Study Group. Dopamine transporter brain imaging to assess the effects of Pramipexole vs levodopa Parkinson disease progression. JAMA. 2002;287: 1653-1661.

31. Parkinson Study Group. Levodopa and the progression of Parkinson disease. N Engl J Med. 2004;351:18-28.

32. Faber TL, Cooke CD, Folks RD, et al. Left ventricular function and perfusion from gated SPECT perfusion images: an integrated method. J Nucl Med. 1999; 40:650-659.

33. Germano G, Kiat H, Kavanagh PB, et al. Automatic quantification of ejection fraction from gated myocardial perfusion SPECT. J Nucl Med. 1995;36:2138-2147.

34. Liu YH, Sinusas AJ, Khaimov D, Gebuza BI, Wackers FJ. New hybrid countand geometry-based method for quantification of left ventricular volumes and ejection fraction from ECG-gated SPECT: methodology and validation. $\mathrm{J} \mathrm{Nucl}$ Cardiol. 2005;12:55-65.

35. Garcia EV, Taylor A, Halkar R, et al. RENEX: an expert system for the interpretation of ${ }^{99 \mathrm{~m}} \mathrm{Tc}-\mathrm{MAG} 3$ scans to detect renal obstruction. J Nucl Med. 2006;47:320-329. 